\title{
External-Beam Radiotherapy Alone Management of Primary CNS Lymphoplasmacytic Lymphoma: A Vietnamese Case Report and Literature Review
}

\author{
Dang Nguyen $\operatorname{Van} \mathbb{1}^{1,2}$ \\ Nghia Duong Van' \\ Quang Le Van' \\ Tung Ngo Thanh ${ }^{2}$ \\ To Ta $\mathrm{Van}^{3}$ \\ 'Department of Oncology, Hanoi Medical \\ University, Hanoi, Vietnam; ${ }^{2}$ Department \\ of Head and Neck Radiation Oncology, \\ Vietnam National Cancer Hospital, \\ Hanoi, Vietnam; ${ }^{3}$ Center of Pathology \\ and Molecular Biology, Vietnam National \\ Cancer Hospital, Hanoi, Vietnam
}

Correspondence: Dang Nguyen Van Department of Oncology, Hanoi Medical University, 0 I Ton That Tung Street, Dong Da District, Hanoi, 10000, Vietnam Tel +84942727899

Email nguyenvandang@hmu.edu.vn; drdangnguyen@gmail.com
Background: Primary central nervous system (CNS) lymphoma is an uncommon nonHodgkin disease limited to the CNS, and most cases are diffuse large B-cell lymphomas. Other pathologies, including lymphoplasmacytic lymphoma (LPL), are exceedingly rare and poorly understood. The clinical presentation of primary CNS LPL is diverse. It depends on the original site and the tumor's extension. There is currently no consensus on a treatment strategy for this uncommon manifestation. To our knowledge, no previously published case was successfully treated with radiation therapy alone.

Case Presentation: We present here a case of primary CNS LPL. A 46-year-old, previously healthy woman was presented with a worsening headache and lower extremity numbness. Multifocal enhanced masses were detected in an MRI with biopsy results consistent with LPL. A complete staging workup was performed with no evidence of systemic disease. The patient received external-beam radiotherapy alone and had a complete remission. After 2 years of follow-up, she remains disease-free.

Conclusion: Radiation alone is a promising treatment option for primary CNS lymphoplasmacytic lymphoma.

Keywords: lymphoplasmacytic lymphoma, primary CNS lymphoma, radiation therapy

\section{Introduction}

Lymphoplasmacytic lymphoma is a low-grade, B-cell neoplasm composed of small lymphocytes, plasmacytoid lymphocytes, and plasma cells that typically involve the bone marrow and it is associated with an immunoglobulin M (IgM) gammopathy. ${ }^{1}$ The diagnosis of LPL is mainly based on the histological evaluation of involved tissue, usually bone marrow or lymph nodes. Although the vast majority of LPL cases are Waldenström macroglobulinemia (WM), there are some exceptions not satisfied with the diagnosis of WM. ${ }^{1}$ They include tumors producing other immunoglobulins, combined immunoglobulins, mixed cryoglobulins, gamma heavy chains, or non-gammopathy disease. ${ }^{2}$ Primary CNS lymphoma is an uncommon variant of non-Hodgkin lymphoma that involves the brain, leptomeninges, spinal cord, or eyes without systemic involvement. In literature, only a few cases diagnosed with LPL in the setting of primary CNS lymphoma have been reported. The current treatment strategy is not consistent due to the rarity and limited knowledge of this entity. 


\section{Case Presentation}

A forty-six-year-old female was presented to the emergency department of our hospital with a two-month-headache and progressive numbness of her right leg. She also complained of nausea and dizziness 2 weeks before admission. The patient's medical and family history revealed no significance. On admission, the patient seemed excitable with a Glasgow Coma Scale (GCS) of 14 (E:4; V:4; M:6); other vital signs were in their normal ranges. In a general examination, no lymphadenopathy or organomegaly was found. A neurological examination revealed numbness in her right lower limb with no motor dysfunction, cranial neuropathy, or signs of increased intracranial pressure. However, magnetic resonance imaging (MRI) of her brain showed multifocal lesions scattered across the left occipital and parietal lobes; the largest one measured $16 \mathrm{~mm}$ in its transverse dimension. These lesions demonstrated a hypointense signal on the T1W sequence, a hyperintense signal on FLAIR, and no abnormal diffusion restriction on DWI images (Figure 1). They also revealed rim enhancement following gadolinium contrast injection.

Concern for brain metastases prompted further diagnostic procedures, including thoracic and abdominal contrast CT, lumbar and thoracic spine MRI, endoscopy of the gastrointestinal tract and ENT, but they identified no abnormalities. The patient then underwent a surgical biopsy with two samples resected from the two largest masses in the left occipital lobe. Microscopically, the lesions were composed of small lymphocytes and neoplasm cells with plasma cell morphological features. The immunohistochemical (IMH) test showed tumor cells are positive for CD79a, CD138, CD38, and MUM-1, but negative for CD3 and CD20 (Figure 2). These microscopic and IMH findings were consistent with lymphoplasmacytic lymphoma. Quantitative determination of the serum globulin test showed an increase in IgE at $740 \mathrm{IU} /$ $\mathrm{mL}$; other globulin levels were in their normal ranges $(\operatorname{IgG}$ 1206 mg/dL, IgM 155 mg/dL, IgA 220 mg/dL, Free Kappa $12.2 \mathrm{mg} / \mathrm{L}$, Free Lambda $12.5 \mathrm{mg} / \mathrm{L}$ ). Her serum protein electrophoresis and serum immunoelectrophoresis tests identified no monoclonal gammopathy. Bone marrow biopsy and cerebrospinal fluid (CSF) analysis detected no cancerous involvement. Pieces of the evidence above confirmed the diagnosis of stage I primary CNS LPL, according to the Arbor-Cotswold staging system for lymphomas.

Since the disease was confined to her brain and she denied every chemotherapy approach, we decided to treat her with whole-brain radiation at a 32 Gy dose (20 fractions of 1,6 Gy per faction). She also received a combination of dexamethasone during radiation therapy. Her headache was relieved after 2 weeks, and the left-foot numbness occasionally only after treatment. Two years after irradiation, clinical examination and imaging workup revealed no neurological deficits or recurrences.

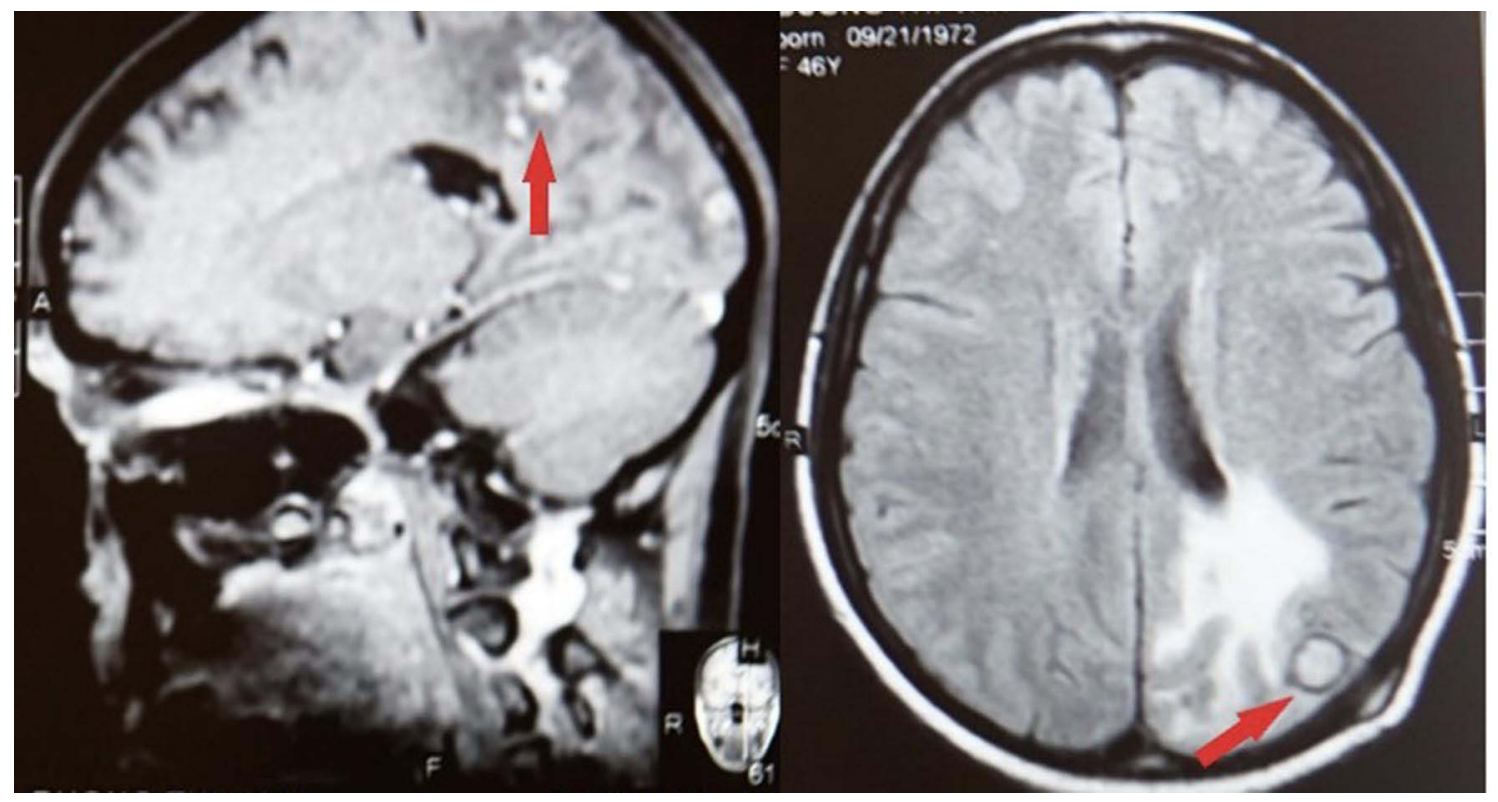

Figure I Contrasted brain MRI revealed enhanced masses in the occipital and parietal lobes (arrow). 


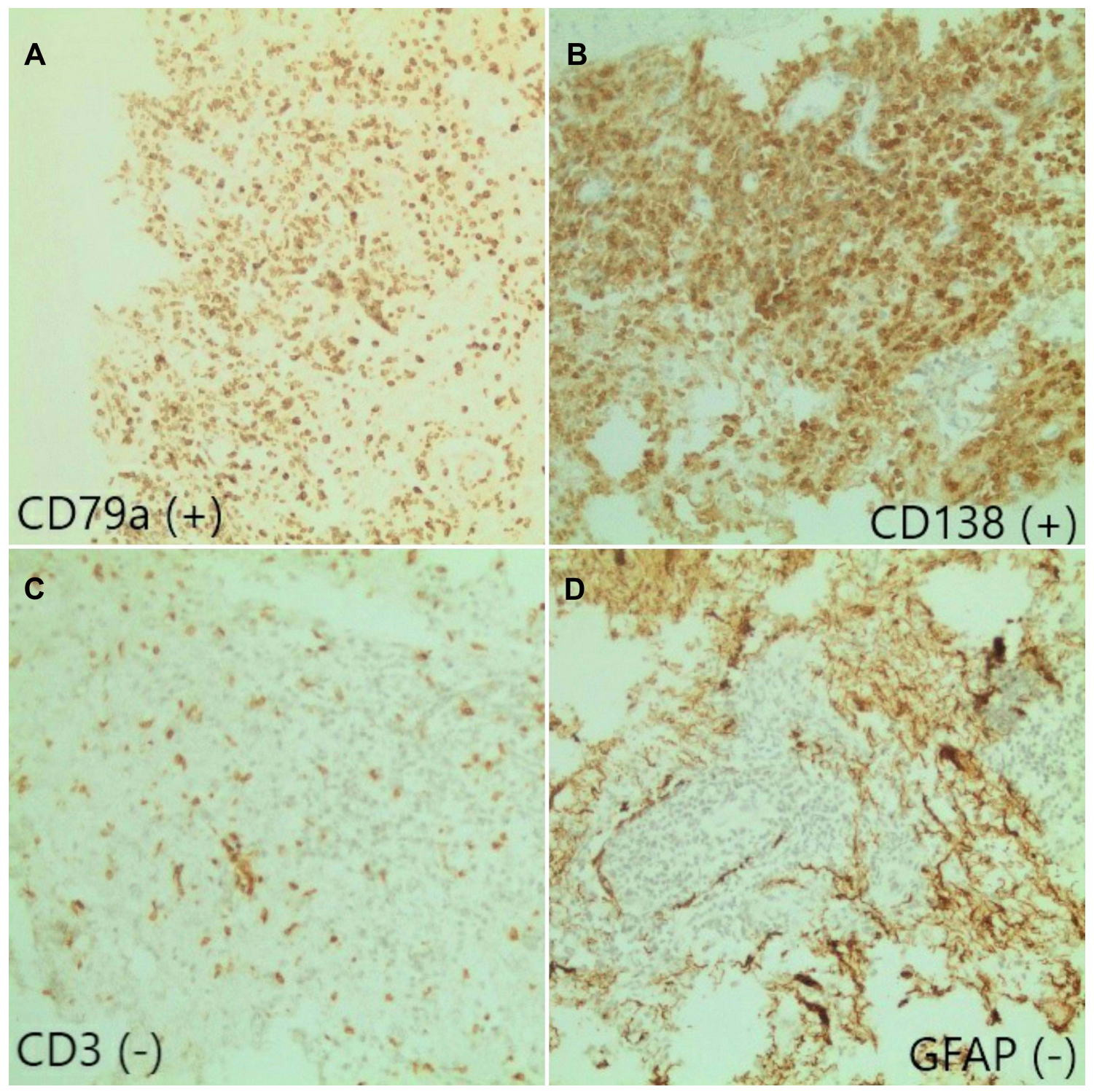

Figure 2 Immunohistochemistry results: (A) CD79a (+); (B) CDI38 (+); (C) CD3 (-); (D) GFAP (-).

\section{Discussion}

Lymphoplasmacytic lymphoma is an uncommon variety of non-Hodgkin lymphoma composed of small lymphocytes, plasmacytoid lymphocytes, and plasma cells. It accounts for about less than $1 \%$ of hematologic malignancies and is even rarer in Asia, with an incidence of $0.31-0.43$ cases per million. ${ }^{3}$ Waldenström macroglobulinemia (WM), the most common subset of LPL, is featured by an elevated serum level of monoclonal immunoglobulin $\mathrm{M}$ and bone marrow involvement. ${ }^{4}$

Primary CNS lymphoma is a very rare subgroup of extranodal non-Hodgkin lymphoma that is confined to the brain parenchyma, leptomeninges, the eye or spinal cord and has no evidence of extra-CNS involvement. Its clinical expression are not specific and vary by site of involvement, including focal neurologic deficits, neuropsychiatric symptoms, signs of increased intracranial pressure, and seizures. Due to the lack of specific symptoms, a definitive diagnosis of primary CNS lymphoma requires a pathological assessment of the involved tissue. Some cases can be diagnosed through the histopathological evaluation of a vitreous biopsy or through a CSF analysis. Diffuse large B-cell lymphoma causes most cases of primary CNS lymphoma, while in rare cases, other entities such as T-cell, lymphoplasmacytic lymphoma and Burkitt's lymphoma are encountered. Primary CNS LPL 
clinical features and optimal treatment remain poorly understood due to the rarity of cases and the difficulty of diagnosis.

In the literature, we found no more than 30 cases of primary CNS LPL published (via a PubMed search for "primary CNS" and "lymphoplasmacytic lymphoma" in case reports). We presented here nine cases with detailed patient information (Table 1); the other cases reported are listed in the references. ${ }^{5-7}$

LPL, like other primary CNS lymphomas, can manifest in the brain, meninges, or spinal cord, but no eye involvement has been reported. However, brain parenchyma is the most common primary site (Table 1). Its clinical expression are atypical and vary by site of involvement, including focal neurologic deficits, neuropsychiatric symptoms, signs of increased intracranial pressure, or seizures. Intracranial masses are usually detected by a head and neck MRI. ${ }^{9}$ In addition, other laboratory assessment tools such as Positron Emission Tomography Scan (PET-CT), blood immunoglobulin tests and bone marrow biopsy are performed to detect other site involvement and exclude WM criteria. Pathological evaluation of tissue, which is essential for confirming LPL diagnosis, typically demonstrates infiltration by small lymphocytes, plasmacytoid lymphocytes, and plasma cells with intranuclear inclusion (Russell bodies) in some reported cases. ${ }^{8}$ The typical immunophenotype of LPL includes expression of CD19, CD22, CD20, CD38, and CD79a and the negative T-cell marker CD3, BCL. Because of limited features clinically and pathologically, LPL needs to be approached as a diagnosis of exclusion.

A major differential diagnosis of primary CNS LPL is Bing-Neel syndrome (BNS). Fintelman et al proposed restricting the definition of BNS to patients with previously confirmed WM and CNS signs not due to hyperviscosity or lymphocyte transformation. ${ }^{16}$ Minnema et al 2017 have provided guidelines for the diagnosis, treatment, and response criteria for Bing-Neel syndrome. It emphasizes the role of cerebral spinal fluid analysis with multiparameter flow cytometry in establishing B-cell clonality, protein electrophoresis and MRI of the brain and spinal cord. ${ }^{17}$ However, bone marrow involvement and $\operatorname{IgM}$ monoclonal paraprotein are essential criteria for WM, but they were omitted in our case, so the diagnosis of primary CNS LPL was established. It is also noted that Bing-Neel syndrome can be diagnosed without fulfilling WM diagnostic criteria. ${ }^{18}$

There is still no consensus guideline for optimal treatment for patients with primary CNS LPL. The chosen therapies in reported cases included tumor resection, chemotherapy, radiation therapy, and targeted therapy. The reviewed case with the best outcome is a 50-year-old patient diagnosed with a mass at the T4 level of the spinal cord. She was treated by complete surgical resection only

Table I Comparison of Clinical Features of Documented Case Reports

\begin{tabular}{|c|c|c|c|c|}
\hline Reference & $\begin{array}{c}\text { Genderl } \\
\text { Age }\end{array}$ & Primary Site & Treatment & Follow-Up \\
\hline Abbi KK et al Case $I^{8}$ & $\mathrm{~F} / 53$ & Left parietal lobe & $\begin{array}{c}\text { Therapeutic Surgery/Tumor bed } \\
\text { RT }\end{array}$ & I year with no recurrence \\
\hline Yan et al Case $2^{9}$ & $\mathrm{~F} / 43$ & Cerebellopontine angle & Total tumor resection/CT & 6 months with no recurrence \\
\hline $\begin{array}{l}\text { Layden et al Case } 3 \\
2011^{10}\end{array}$ & $M / 50$ & Hypothalamic region & Tumor biopsy/CT & 6 months of stable disease \\
\hline Ikeda et al Case $4^{\text {II }}$ & $\mathrm{F} / 43$ & $\begin{array}{l}\text { Hypothalamus and optic } \\
\text { chiasm }\end{array}$ & & \\
\hline Lim T et al Case $5^{12}$ & $M / 50$ & T4 spinal cord & Total tumor resection & $\begin{array}{l}\text { Alive in remission after } 5 \mathrm{I} \\
\text { months }\end{array}$ \\
\hline Lim Tet al Case $6^{12}$ & $M / 38$ & Corpus callosum & Tumor biopsy/CT & 2.6 months \\
\hline Braks et al Case $7^{13}$ & $\mathrm{~F} / 42$ & Right centrum semiovale & Tumor biopsy/ CT + intrathecal & 6 months with no recurrence \\
\hline Carrasco et al Case $8^{14}$ & $\mathrm{~F} / 49$ & Sellar and suprasellar region & Total tumor resection & 4 years with no recurrence \\
\hline Kanavaros et al Case $9^{15}$ & $F / 59$ & Corpus callosum & Tumor biopsy & \\
\hline
\end{tabular}


(corpectomy) and lived without neurological sequelae and had no relapse after 51 months. Interestingly, in the treatment of primary CNS lymphoma, the majority of which is DLBCL, surgery has a limited role because the primary CNS lymphoma nature tends to be systemic rather than localized. Up to now, high-dose intravenous methotrexate (MTX) is the most effective agent against primary CNS lymphoma. In contrast, other combined regimens used in treating systemic lymphoma are either ineffective or intolerable.

Radiation therapy used to be used as a single-modality regimen to treat primary CNS lymphoma in the $70 \mathrm{~s} .{ }^{19}$ Nowadays, radiation therapy plays a restricted role in primary CNS lymphoma and is only used as a consolidation modality after induction of MTX or as a salvage treatment after disease recurrence or persistence. However, primary CNS lymphoma is a radiosensitive disease, with over $90 \%$ of patients achieving a response and symptoms relieved in $84 \% .{ }^{20}$ For patients who are unsuitable or unwilling to engage in chemotherapy, whole-brain radiation is an acceptable alternative, despite the higher risk of disease progression and late neurotoxicity. Since low-grade B-cell lymphomas, including LPL, have a better prognosis than diffuse large B-cell lymphoma, radiation therapy alone is also a promising pathway, besides chemotherapy and surgical resection. One of the late toxicity of whole-brain radiation therapy is damage to the hippocampus. However, with the advancement of radiotherapy techniques today, it is possible to minimize the late toxicity of whole-brain radiation therapy. ${ }^{21}$ Further investigation and randomized trials should be executed to confirm the effect and validate the optimal radiotherapy dose.

\section{Conclusion}

Primary CNS lymphoplasmacytic lymphoma is an uncommon variety of non-Hodgkin lymphoma very similar to the Bing - Neel syndrome. The definitive diagnosis is mainly based on pathology and immunohistochemistry. Currently, the treatment strategy is not uniform but individualized depending on each case. We report a clinical case of a patient with primary CNS lymphoplasmacytic lymphoma, and was treated with radiation therapy alone. We suggest that radiotherapy may be an encouraging strategy for this disease.

\section{Abbreviations}

CNS, central nervous system; LPL, lymphoplasmacytic lymphoma; WM, Waldenström macroglobulinemia; IMH, immunohistochemical; CSF, cerebrospinal fluid; BNS, Bing-Neel syndrome; MTX, methotrexate.

\section{Data Sharing Statement}

The datasets used in the current study are available upon reasonable request from the corresponding author.

\section{Ethics Approval and Informed Consent}

The study was approved by our research committee, Hanoi Medical University, Hanoi, Vietnam and Vietnam National Cancer Hospital, Hanoi, Vietnam.

\section{Consent for Publication}

The publication of this study has been consented to by the patient.

\section{Author Contributions}

All authors contributed to data analysis, drafting or revising the article, gave final approval of the version to be published, agreed to the submitted journal, and agree to be accountable for all aspects of the work.

\section{Funding}

There is no funding to report.

\section{Disclosure}

The authors report no conflicts of interest in this work.

\section{References}

1. Naderi N, Yang DT. Lymphoplasmacytic lymphoma and Waldenström macroglobulinemia. Arch Pathol Lab Med. 2013;137(4):580-585. doi:10.5858/arpa.2012-0034-RS

2. Marzia Varettoni, Emanuela Boveri, Silvia Zibellini, et al. Clinical and molecular characteristics of lymphoplasmacytic lymphoma not associated with an IgM monoclonal protein: A multicentric study of the Rete Ematologica Lombarda (REL) network. Am J Hematol. 2019;94 (11):1193-1199. doi: 10.1002/ajh.25600

3. Iwanaga M, Chiang C-J, Soda M, et al. Incidence of lymphoplasmacytic lymphoma/Waldenström's macroglobulinaemia in Japan and Taiwan population-based cancer registries, 1996-2003. Int J Cancer. 2014;134(1):174-180. doi:10.1002/ijc.28343

4. Swerdlow SH, Campo E, Harris NL, et al. WHO Classification of Tumours of Haematopoietic and Lymphoid Tissues. 4th ed. Lyon, France: International agency for research on cancer; 2008.

5. Jahnke K, Korfel A, O'Neill BP, et al. International study on low-grade primary central nervous system lymphoma. Ann Neurol. 2006;59(5):755-762. doi:10.1002/ana.20804

6. Bogdahn U, Bogdahn S, Mertens HG, et al. Primary non-Hodgkin's lymphomas of the CNS. Acta Neurol Scand. 1986;73(6):602-614. doi:10.1111/j.1600-0404.1986.tb04607.x 
7. Shenkier TN. Unusual variants of primary central nervous system lymphoma. Hematol Oncol Clin North Am. 2005;19(4):651-664. doi:10.1016/j.hoc.2005.05.001

8. Abbi KKS, Muzaffar M, Gaudin D, et al. Primary CNS lymphoplasmacytic lymphoma: a case report and review of literature. Hematol Oncol Stem Cell Ther. 2013;6(2):76-78. doi:10.1016/j.hemonc.2013.06.002

9. Yan C, Kong X, Yang L, et al. An uncommon case of lymphoplasmacytic lymphoma in cerebellopontine angle region. Medicine (Baltimore). 2016;95(34):e4627. doi:10.1097/MD.0000000000004627

10. Layden BT, Dubner S, Toft DJ, et al. Primary CNS lymphoma with bilateral symmetric hypothalamic lesions presenting with panhypopituitarism and diabetes insipidus. Pituitary. 2011;14(2):194-197. doi:10.1007/s11102-008-0166-7

11. Ikeda T, Hara K, Yamanaka T, et al. [A case of primary central nervous system malignant lymphoma developing from the optic chiasma and hypothalamus]. Rinsho Shinkeigaku. 2006;46 (7):475-479. Japanese.

12. Lim T, Kim SJ, Kim K, et al. Primary CNS lymphoma other than DLBCL: a descriptive analysis of clinical features and treatment outcomes. Ann Hematol. 2011;90(12):1391-1398. doi:10.1007/ s00277-011-1225-0

13. Braks E, Urbach H, Pels H, et al. Primary central nervous system immunocytoma: MRI and spectroscopy. Neuroradiology. 2000;42:738-741. doi:10.1007/s002340000392

14. Carrasco CA, Rojas ZD, Chiorino R, et al. Primary pituitary lymphoma in immunocompetent patient: diagnostic problems and prolonged follow-up. Pituitary. 2012;15(1):93-96. doi:10.1007/s11102010-0219-6
15. Kanavaros P, Mikol J, Nemeth J, et al. Stereotactic biopsy diagnosis of primary non-Hodgkin's lymphoma of the central nervous system: a histological and immunohistochemical study. Pathol Res Pract. 1990;186(4):459-466. doi:10.1016/S0344-0338(11)80464-4

16. Fintelmann F, Forghani R, Schaefer PW, et al. Bing-Neel syndrome revisited. Clin Lymphoma Myeloma. 2009;9(1):104-106. doi:10.3816/CLM.2009.n.028

17. Minnema MC, Kimby E, D'Sa S. Guideline for the diagnosis, treatment and response criteria for Bing-Neel syndrome. Haematologica. 2017;102(1):43-51. doi:10.3324/haematol.2016.147728

18. Grainger BT, Issa S. Bing-Neel syndrome presenting as isolated CNS lymphoplasmacytic lymphoma: a case report and review of the literature. J Clin Neurosci. 2019;71:277-280.

19. Henry JM, Heffner RR, Dillard SH, et al. Primary malignant lymphomas of the central nervous system. Cancer. 1974;34 (4):1293-1302. doi:10.1002/1097-0142(197410)34:4<1293::AIDCNCR2820340441>3.0.CO;2-P

20. Kwak Y-K, Choi B-O, Choi KH, et al. Radiotherapy as an alternative treatment option for primary central nervous system lymphoma patients who are noncandidates for chemotherapy. Oncotarget. 2017;8(63):106858-106865. doi:10.18632/oncotarget.22427

21. Kazda T, Pospíšil P, Doleželová H, et al. Whole brain radiotherapy: consequences for personalized medicine. Rep Pract Oncol Radiother. 2013;18(3):133-138. doi:10.1016/j.rpor.2013.03.002
Journal of Blood Medicine

\section{Publish your work in this journal}

The Journal of Blood Medicine is an international, peer-reviewed, open access, online journal publishing laboratory, experimental and clinical aspects of all aspect pertaining to blood based medicine including but not limited to: Transfusion Medicine; Blood collection, Donor issues, Transmittable diseases, and Blood banking logistics; Immunohematology; Artificial and alternative blood based

\section{Dovepress}

therapeutics; Hematology; Biotechnology/nanotechnology of blood related medicine; Legal aspects of blood medicine; Historical perspectives. The manuscript management system is completely online and includes a very quick and fair peer-review system. Visit http://www.dovepress.com/testimonials.php to read real quotes from published authors. 